\title{
A importância da prevenção dos fatores de riscos no acidente vascular encefálico:
}

\section{revisão integrativa da literatura}

\author{
The importance of preventing risk factors in stroke: integrative literature review \\ La importancia de prevenir los factores de riesgo en el accidente cerebrovascular: revisión \\ integradora de la literatura
}

Recebido: 02/12/2021 | Revisado: 07/12/2021 | Aceito: 08/12/2021 | Publicado: 17/12/2021

\author{
Melquisedeque Silva de Oliveira \\ ORCID: https://orcid.org/0000-0003-3799-6983 \\ Centro Universitário da Amazônia, Brasil \\ e-mail: melqui.chelsea.28@gmail.com \\ Regiane de Paula Maciel \\ ORCID: https://orcid.org/0000-0002-4072-085X \\ Centro Universitário da Amazônia, Brasil \\ e-mail: regianestm@ hotmail.com
}

\begin{abstract}
Resumo
O Acidente Vascular Encefálico (AVE) é uma doença caracterizada por um comprometimento funcional neurológico, envolvendo o sistema nervoso central resultando em um distúrbio de circulação. O mesmo pode ser qualificado por isquêmico, ao acontecer a obstrução dos vasos sanguíneos ou hemorrágico originado através da ruptura de um vaso sanguíneo. Objetiva-se enfatizar a importância da prevenção dos fatores de risco no Acidente Vascular Encefálico, a partir de uma revisão integrativa da literatura. A pesquisa direcionou o foco para uma perspectiva dos métodos de revisão integrativa da literatura. Coleta de dados dos artigos na literatura foi realizada uma busca nas seguintes bases de dados: Literatura Latino-Americana e do Caribe em Ciências da Saúde (LILACS) e Medical Literature Analysis and Retrieval Sistem on-line (Medline), Google acadêmico e Scielo. O risco de AVE começa a aumentar com o passar dos anos, Entre os principais fatores de risco, existem fatores não modificáveis que são: a hereditariedade, o sexo e a etnia, sendo o sexo masculino e a raça negra que apresentam maior incidência de AVE isquêmica. Enquanto nos fatores de risco modificáveis, se classificam a hipertensão arterial, sendo o principal deles, onde acarreta um aumento superior a três vezes na incidência em casos de AVE. Conclui-se que o diagnóstico precoce assegura maior minimização dos danos ao paciente e também é de responsabilidade da equipe de saúde e do enfermeiro.
\end{abstract}

Palavras-chave: Acidente cerebral vascular; Hipertensão; Acidentes cerebrovasculares.

\begin{abstract}
The Cerebrovascular Accident (CVA) is a disease characterized by a functional neurological impairment, involving the central nervous system resulting in a circulation disorder. The same can be qualified as ischemic, when there is obstruction of blood vessels or hemorrhagic caused by the rupture of a blood vessel. The objective is to emphasize the importance of preventing risk factors in Stroke, based on an integrative literature review. The research focused on a perspective of integrative literature review methods. Data collection of articles in the literature was performed a search in the following databases: Latin American and Caribbean Literature in Health Sciences (LILACS) and Online Medical Literature Analysis and Retrieval Sistem (Medline), Academic Google and Scielo. The risk of stroke begins to increase over the years. Among the main risk factors, there are non-modifiable factors which are: heredity, sex and ethnicity, with males and blacks having a higher incidence of stroke ischemic. As for modifiable risk factors, hypertension is classified, being the main one, which causes an increase of more than three times in the incidence in cases of stroke. It is concluded that early diagnosis ensures greater minimization of harm to the patient and is also the responsibility of the health team and nurses.
\end{abstract}

Keywords: Stroke; Hypertension; Cerebrovascular accidents.

\section{Resumen}

El Accidente Cerebrovascular (ACV) es una enfermedad caracterizada por un deterioro neurológico funcional, que involucra al sistema nervioso central y resulta en un trastorno de la circulación. Lo mismo puede calificarse de isquémico, cuando existe obstrucción de los vasos sanguíneos o hemorrágico provocado por la rotura de un vaso sanguíneo. El objetivo es enfatizar la importancia de prevenir los factores de riesgo en el ictus, a partir de una revisión integradora de la literatura. La investigación se centró en una perspectiva de métodos integradores de revisión de la literatura. La recolección de datos de artículos en la literatura se realizó una búsqueda en las siguientes bases de datos: Literatura Latinoamericana y del Caribe en Ciencias de la Salud (LILACS) y Sistema de Análisis y Recuperación de 
Literatura Médica en Línea (Medline), Google Académico y Scielo. El riesgo de ictus comienza a aumentar con los años, entre los principales factores de riesgo se encuentran los factores no modificables que son: la herencia, el sexo y la etnia, siendo los hombres y los negros los que tienen una mayor incidencia de ictus isquémico. En cuanto a los factores de riesgo modificables, se clasifica la hipertensión, siendo el principal, la que provoca un aumento de más de tres veces en la incidencia en los casos de ictus. Se concluye que el diagnóstico precoz asegura una mayor minimización del daño al paciente y también es responsabilidad del equipo de salud y enfermeras.

Palabras clave: Accidente cerebrovascular; Hipertensión; Accidentes cerebrovasculares.

\section{Introdução}

O Acidente Vascular encefálico (AVE) é uma doença caracterizada por um comprometimento funcional neurológico, envolvendo o sistema nervoso central resultando em um distúrbio de circulação. O início abrupto ou em forma de crise com sintomas neurológicos focais ou globais, causados por isquemia ou hemorragia no cérebro ou a sua volta, como, um déficit neurológico focal súbito devido a uma lesão vascular (Barnett e Spence, 2016).

No decorrer do trabalho será abordado o funcionamento da fisiologia cerebral e a sua patologia quando o cérebro é acometido no funcionamento, podendo envolver o estado físico e mental da pessoa. $\mathrm{O}$ acidente vascular encefálico pode ser classificado como, acidente vascular encefálico isquêmico (AVEI) (decorrente de uma interrupção da circulação cerebral) ou acidente vascular encefálico hemorrágico (AVEH) (ruptura de um vaso sanguíneo) (Nunes et al. 2017).

Nos dias de hoje, o AVE vem se tornando uma das patologias mais comum. Contudo a incidência entre adultos e jovens são raras e seu diagnóstico se constitui um desafio clínico. As causas são diversas, sendo que as vítimas mais acometidas são da idade idosa (Loft et al. 2017).

Conforme Silva et al. (2020b), há determinados fatores de risco para os acidentes cerebrovasculares estão qualificados em dois grupos: os fatores de risco não modificáveis: os de maior expressão é a idade sendo que a incidência cresce excepcionalmente após os 65 anos e quanto ao gênero, estudos explanam que a incidência de AVE é comum em homens e entre afro-americanos.

Nos fatores de risco modificáveis; estão incluídas a Hipertensão Arterial Sistêmica (HAS), ponderada como o potencial de risco para o acidente cerebral vascular, no qual pessoas que possuem o HAS têm mais facilidade de desenvolver o AVE. O HAS pode ser culpado por até a metade dos casos de AVS (Silva et al. 2020b).

A atuação do profissional da saúde frente a esta situação requer muita atenção, pois a assistência de enfermagem deve ser prestada com qualidade e cuidado ao paciente com suspeita, investigando-o de forma cautelosa para se obter resultados precisos e certos, pois uma vez dado o diagnóstico incorreto o prejuízo pode ser grande (Oliveira et al. 2018).

$\mathrm{O}$ atendimento também é extensivo aos familiares no qual a equipe de saúde passa orientações aos familiares sobre a, evolução, quadro clínico, cuidados a serem tomados e junto a isso oferecendo apoio emocional e encontrar a melhor maneira possível de enfrentar e vivenciar a doença que se interpôs no caminho de uma meta de sua vida (Cruz, 2016).

Os cuidados pelos profissionais de enfermagem na prevenção de fatores de riscos que levam ao Acidente Vascular Encefálico podem reduzir o número de indivíduos com AVE e com isso reduzir o número de mortes e diminuir a ocupação hospitalar (Rodrigues et al. 2019).

O acidente vascular encefálico é uma das principais causas de mobilidade e mortalidade no mundo todo apresentando uma grande incidência, acometendo a sociedade em geral, independente das condições socioeconômicas. Sendo causado por diversos fatores, tanto em países desenvolvidos como nos subdesenvolvidos (Lima et al. 2016).

Muita coisa que antes era necessário sair de casa, hoje com a tecnologia, deixou de ser necessário, antigamente uma pessoa se movimentava bem mais, desde a ir ao supermercado ou ir até a farmácia comprar um remédio, agora basta acessar o aplicativo no celular, até mesmo para ligar a televisão ou mudar de canal, basta apenas a pessoa utilizar o controle remoto, com 
isso a população está ficando cada vez mais sedentária e adquirir doenças que antes era mais difícil de adquirir, como o AVE (Oliveira et al. 2018).

E ao estudar esse tema, é possível entender a importância do profissional de enfermagem na prevenção de fatores de riscos que levam ao AVE, e com isso diminuir o número de pessoas acometidas com o AVE (Barnett \& Spence, 2016).

O AVE é caracterizado por uma incapacitação súbita da circulação cerebral causada por um bloqueio parcial ou total de um ou mais vasos sanguíneos cerebrais. Quando as células cerebrais (neurônios) são desprovidas de oxigênio por poucos minutos, ocorre a destruição celular e a morte do tecido cerebral (infarto cerebral). Os sintomas são muito variáveis e dependem da área específica afetada (Zukerman, 2016).

Com a tecnologia presente, praticamente tudo pode ser feito por meio digital, com isso as pessoas foram ficando cada vez mais sedentária e com isso passou a aumentar o número de AVE (Cruz, 2016). Com isso pergunta-se, a assistência dos profissionais de enfermagem na prevenção de fatores de riscos que levam ao Acidente Vascular Encefálico?

O objetivo do presente trabalho é enfatizar a importância da prevenção dos fatores de risco no Acidente Vascular Encefálico, a partir de uma revisão integrativa da literatura e os objetivos específicos foram: conceituar e classificar o Acidente Vascular Encefálico, descrever os fatores de risco do Acidente Vascular Encefálico e enfatizar o papel do enfermeiro na prevenção dos fatores de risco do Acidente Vascular Encefálico.

\section{Metodologia}

A pesquisa direcionou o foco para uma perspectiva dos métodos de revisão integrativa da literatura. Os quais se fundamentam principalmente em análise, pela não utilização de instrumental estatístico de análise de dados. Esse tipo de análise tem por base conhecimentos teórico-empíricos que permite atribuir-lhe cientificidade (Souza et al. 2010). Tais dados foram empregados de diferentes técnicas; estudos divulgados com a finalidade de descrever os fatos, seus significados, buscando as respostas dos fenômenos do mundo social.

O desenvolvimento aconteceu de forma exploratória/descritiva a fim de proporcionar uma aproximação ao tema. Este tipo de pesquisa tem a finalidade de ampliar o conhecimento a respeito de determinado fenômeno. Em sua obra Fernandes et al. (2018) corroboram que a pesquisa descritiva se constitui um estudo que é intermediário entre a pesquisa exploratória e a explicativa, ou seja, não é tão superficial como a primeira e nem tão profunda como a segunda. Ao unir os dois métodos, neste estudo, foi possível ter uma melhor análise do tema proposto.

Diante disso a descrição significa: fazer comparação, relatar, identificar. Por isso é realizado levando em conta as questões da formulação das perguntas que conduzem a pesquisa, além de estabelecer uma relação entre as variáveis propostas no objeto de estudo em análise. As fontes de informações na elaboração da pesquisa foram as bibliográficas, as quais têm como base os materiais disponibilizados na Internet: artigos, publicações e periódicos.

Foi realizada uma busca nas seguintes bases de dados: Literatura Latino-Americana e do Caribe em Ciências da Saúde (LILACS) e Medical Literature Analysis and Retrieval Sistem on-line (Medline), Google acadêmico e Scielo.

Os critérios de inclusão definidos para a seleção dos artigos foram: artigos publicados em português e inglês; artigos na íntegra que retratassem a temática referente ao estudo e artigos publicados e indexados nos referidos bancos de dados no período de 2016 a 2021. Foram excluídos: trabalhos incompletos, com informações apenas em resumos e que não atendessem aos critérios de inclusão.

Para determinar a amostra do estudo foram selecionados os seguintes descritores em saúde: acidente cerebral vascular, hipertensão, acidentes cerebrovasculares. Tornando possível escolher de forma mais adequada os artigos que respondessem ao objetivo proposto. Num momento posterior, realizou-se leitura extensa do material selecionado, retirando os conceitos abordados e de interesse, segundo o objetivo deste estudo. 
Após a coleta de dados da pesquisa, foi realizada a análise e interpretação destes. A análise e a interpretação das informações têm como objetivo estabelecer e sumarizar os dados de maneira que permitam encontrar as respostas do problema proposto. Já a interpretação objetiva a procurar do sentido mais amplo das respostas.

As informações dos trabalhos selecionados foram sumarizadas utilizando o programa Excel, em uma ficha de extração de dados denominada Identificação e exposição do conteúdo do artigo que aponta os seguintes itens: tema, título, autores, fonte da publicação, objetivo, coleta das informações/tipos de pesquisa, análise das informações, resultados/discussão e conclusões.

Para a análise de dados deste estudo, foi utilizada como suporte o método de análise de conteúdo proposto por Bardin (2012), o método trata -se de um compilado de técnicas de análise de comunicações, com a finalidade de descrever conteúdos e mensagens, utilizando -se de processos sistemáticos. Para melhor compreensão deste método, Azevedo et al. (2021), sintetizou as fases de análise de dados em sua obra.

Figura 1. Resumo do método de análise de conteúdo de Bardin.

\section{MÉTODO DE ANÁLISE DE CONTEÚDO}

\begin{tabular}{c|l}
\hline $\begin{array}{c}\text { FASE I } \\
\text { Pré - análise }\end{array}$ & $\begin{array}{l}\text { Seleção dos materiais que tem relação com o objeto de estudo, embasamento argumentativos } \\
\text { para os resultados obtidos. Nesta fase são aplicados os critérios de inclusão e exclusão, para } \\
\text { facilitar a obtenção de dados que serão realmente aproveitados. }\end{array}$ \\
\hline $\begin{array}{c}\text { FASE II } \\
\text { Exploração do } \\
\text { material }\end{array}$ & $\begin{array}{l}\text { Estudo e leitura minuciosa dos materiais obtidos. Nesta fase são avaliação os escritos e } \\
\text { selecionados os que serão necessários para sustentar do tema de estudo, assim como os que } \\
\text { serão utilizados para fazer correlação com a proposta do tema em questão. }\end{array}$ \\
\hline $\begin{array}{c}\text { FASE III } \\
\text { Tratamento dos } \\
\text { resultados }\end{array}$ & $\begin{array}{l}\text { Validação dos dados analisados. Relação dos objetivos com os resultados, e possível } \\
\text { surgimento de novas hipóteses. }\end{array}$ \\
\hline
\end{tabular}

Fonte: Azevedo et al (2021).

\section{Resultados e Discussão}

Conforme Silva et al. (2020), há determinados fatores de risco para os acidentes cerebrovasculares estão qualificados em dois grupos: os fatores de risco não modificáveis: os de maior expressão é a idade sendo que a incidência cresce excepcionalmente após os 65 anos e quanto ao gênero, estudos explanam que a incidência de AVE é comum em homens e entre afro-americanos.

Nos fatores de risco modificáveis; estão incluídas a Hipertensão Arterial Sistêmica (HAS), ponderada como o potencial de risco para o acidente cerebral vascular, no qual pessoas que possuem o HAS têm mais facilidade de desenvolver o AVE. O HAS pode ser culpado por até a metade dos casos de AVS (Silva et al. 2020b).

A atuação do profissional da saúde frente a esta situação requer muita atenção, pois a assistência de enfermagem deve ser prestada com qualidade e cuidado ao paciente com suspeita, investigando-o de forma cautelosa para se obter resultados precisos e certos, pois uma vez dado o diagnóstico incorreto o prejuízo pode ser grande (Oliveira et al. 2018).

Os resultados mostram que vários fatores interferem no cuidado de enfermagem a pacientes com acidente vascular encefálico, sendo que a redução de permanência hospitalar do paciente, acompanhada pelo aumento dos números de internações de pacientes com a doença e pela redução do quadro de pessoal foram as que obtiveram maior relevância.

Portanto que o enfermeiro tem um papel extremamente importante no cuidado de pacientes acometidos pelo AVE, tanto na sua recuperação e adaptação a nova rotina de vida por meio de suas sequelas. A seguir segue os resultados encontrados no Quadro 1. 
Quadro 1. Artigos selecionados.

\begin{tabular}{|c|c|c|c|c|c|}
\hline Item & Autor/ Ano & Tema & Objetivos & Metodologia & Resultados e Conclusões \\
\hline A1 & $\begin{array}{c}\text { Lima et al. } \\
2016\end{array}$ & $\begin{array}{l}\text { Diagnostico de enfermagem } \\
\text { em pacientes com acidente } \\
\text { Vascular cerebral: revisão } \\
\text { integrativa. }\end{array}$ & $\begin{array}{l}\text { Verificar os diagnósticos de } \\
\text { enfermagem presentes nos } \\
\text { pacientes acometidos por } \\
\text { AVC. }\end{array}$ & $\begin{array}{l}\text { Revisão } \\
\text { integrativa da } \\
\text { literatura. }\end{array}$ & $\begin{array}{l}\text { Percebeu-se um enfoque das publicações } \\
\text { nos diagnósticos de enfermagem } \\
\text { relacionados aos distúrbios motores, } \\
\text { como risco de quedas e mobilidade física } \\
\text { prejudicada. Os domínios relacionados a } \\
\text { segurança / proteção (domínio 11) e ao } \\
\text { sono / repouso (domínio 4) estiveram } \\
\text { presentes na maior parte das publicações } \\
\text { avaliadas. }\end{array}$ \\
\hline A2 & $\begin{array}{l}\text { Aleluia } \\
\text { et al. } 2017\end{array}$ & $\begin{array}{l}\text { Coordenação do cuidado na } \\
\text { atenção primaria à saúde: } \\
\text { estudo avaliativo em município } \\
\text { sede de macrorregião do } \\
\text { nordeste brasileiro. }\end{array}$ & $\begin{array}{l}\text { Analisar a coordenação de } \\
\text { atenção pela atenção } \\
\text { primaria à saúde (SPS) em } \\
\text { um sistema municipal de } \\
\text { saúde do Estado da Bahia. }\end{array}$ & $\begin{array}{l}\text { Revisão da } \\
\text { literatura. }\end{array}$ & $\begin{array}{l}\text { Os resultados e a estrutura conceitual } \\
\text { para avaliar a coordenação de cuidados } \\
\text { são contribuição relevante para este } \\
\text { estudo, que pode ser aplicado a outros } \\
\text { contextos com características } \\
\text { semelhantes. }\end{array}$ \\
\hline A3 & $\begin{array}{l}\text { Loft et al. } \\
2017\end{array}$ & $\begin{array}{l}\text { Fortalecendo o papel e as } \\
\text { funções da equipe de } \\
\text { enfermagem na Reabilitação } \\
\text { do acidente vascular encefálico } \\
\text { em pacientes internados: O } \\
\text { desenvolvimento de uma } \\
\text { intervenção complexa usando a } \\
\text { roda de mudança de } \\
\text { Comportamento. }\end{array}$ & $\begin{array}{l}\text { Descrever o } \\
\text { desenvolvimento de uma } \\
\text { intervenção de enfermagem } \\
\text { com o objetivo de otimizar a } \\
\text { reabilitação hospitalar de } \\
\text { pacientes com AVC, } \\
\text { fortalecendo o papel e as } \\
\text { funções da equipe de } \\
\text { enfermagem. }\end{array}$ & $\begin{array}{l}\text { Revisão } \\
\text { sistemática. }\end{array}$ & $\begin{array}{l}\text { Os resultados mostram uma análise } \\
\text { comportamental para explicar por que a } \\
\text { equipe de enfermagem estava ou não } \\
\text { engajada nestes comportamentos. A } \\
\text { capacidade, oportunidade e motivação da } \\
\text { equipe de enfermagem, foram analisadas } \\
\text { no que diz respeito a trabalhar } \\
\text { sistematicamente com uma abordagem } \\
\text { reabilitadora e trabalhar deliberada e } \\
\text { sistematicamente com os objetivos do } \\
\text { paciente. }\end{array}$ \\
\hline A4 & $\begin{array}{c}\text { Nunes et al. } \\
2017\end{array}$ & $\begin{array}{l}\text { Cuidado de enfermagem ao } \\
\text { paciente vítima de acidente } \\
\text { vascular encefálico. }\end{array}$ & $\begin{array}{l}\text { Investigar as intervenções } \\
\text { de enfermagem aos } \\
\text { pacientes com acidente } \\
\text { vascular encefálico no } \\
\text { âmbito hospitalar. }\end{array}$ & $\begin{array}{l}\text { Revisão da } \\
\text { literatura. }\end{array}$ & $\begin{array}{l}\text { Os resultados mostram que vários fatores } \\
\text { interferem no cuidado de enfermagem a } \\
\text { pacientes com acidente vascular } \\
\text { encefálico, sendo que a redução de } \\
\text { permanência hospitalar do paciente, } \\
\text { acompanhada pelo aumento dos números } \\
\text { de internações de pacientes com a } \\
\text { doença e pela redução do quadro de } \\
\text { pessoal foram as que obtiveram maior } \\
\text { relevância. }\end{array}$ \\
\hline A5 & $\begin{array}{c}\text { Santana e } \\
\text { Chun, } 2017\end{array}$ & $\begin{array}{l}\text { Linguagem e funcionalidade de } \\
\text { adultos pós-acidente vascular } \\
\text { encefálico (AVE): Avaliação } \\
\text { baseada na classificação } \\
\text { internacional de } \\
\text { funcionalidade, incapacidade e } \\
\text { saúde (CIF). }\end{array}$ & $\begin{array}{l}\text { Avaliar e classificar } \\
\text { aspectos de linguagem, } \\
\text { funcionalidade e } \\
\text { participação de pessoas pós- } \\
\text { acidente vascular encefálico } \\
\text { com base conceitual da } \\
\text { classificação internacional } \\
\text { de Funcionalidade, } \\
\text { incapacidade e saúde e } \\
\text { caracterizar o perfil sócio } \\
\text { demográfico dos } \\
\text { participantes. }\end{array}$ & $\begin{array}{l}\text { Pesquisa do tipo } \\
\text { transversal } \\
\text { descritiva. }\end{array}$ & $\begin{array}{l}\text { Os resultados mostram o impacto da } \\
\text { dificuldade de linguagem nas vidas das } \\
\text { pessoas pós-acidente vascular encefálico } \\
\text { e participação numa abordagem integral } \\
\text { e humanizada, em prol do } \\
\text { aprimoramento da assistência à saúde no } \\
\text { atendimento ambulatório desse grupo. }\end{array}$ \\
\hline A6 & $\begin{array}{c}\text { Oliveira } \\
\text { et al. } 2018\end{array}$ & $\begin{array}{l}\text { O papel do enfermeiro no } \\
\text { cuidado a pacientes acometidos } \\
\text { por acidente vascular } \\
\text { encefálico. }\end{array}$ & $\begin{array}{l}\text { Descrever o papel de o } \\
\text { enfermeiro no cuidar em } \\
\text { enfermagem ao paciente } \\
\text { acometido por acidente } \\
\text { vascular encefálico. }\end{array}$ & $\begin{array}{l}\text { Revisão } \\
\text { integrativa. }\end{array}$ & $\begin{array}{l}\text { Conclui-se, portanto que o enfermeiro } \\
\text { tem um papel extremamente importante } \\
\text { no cuidado de pacientes acometidos pelo } \\
\text { AVE, tanto na sua recuperação e } \\
\text { adaptação a nova rotina de vida por meio } \\
\text { de suas sequelas. }\end{array}$ \\
\hline A7 & $\begin{array}{l}\text { Dias et al. } \\
2019\end{array}$ & $\begin{array}{l}\text { Utilização de monofilamentos } \\
\text { para avaliação sensorial em } \\
\text { pacientes com sequela de } \\
\text { acidente vascular encefálico } \\
\text { (AVE) - uma revisão } \\
\text { sistemática. }\end{array}$ & $\begin{array}{l}\text { Verificar a utilização dos } \\
\text { monofilamentos como } \\
\text { instrumento de avaliação da } \\
\text { sensibilidade de pacientes } \\
\text { com sequela de AVE na } \\
\text { literatura. }\end{array}$ & $\begin{array}{l}\text { Revisão } \\
\text { sistemática. }\end{array}$ & $\begin{array}{l}\text { Conclui-se que por mais promissor que } \\
\text { seja o uso dos monofilamentos para } \\
\text { avaliação sensorial, esta revisão mostrou } \\
\text { que existem poucos estudos avaliando os } \\
\text { pacientes com sequelas de AVE com } \\
\text { este método de avaliação, principalmente } \\
\text { estudos clínicos. }\end{array}$ \\
\hline
\end{tabular}




\begin{tabular}{|l|l|l|l|l|l|}
\hline A8 & $\begin{array}{l}\text { Rodrigues } \\
\text { et al. 2019 }\end{array}$ & $\begin{array}{l}\text { O conhecimento do enfermeiro } \\
\text { e sua Atuação no acidente } \\
\text { vascular encefálico. }\end{array}$ & $\begin{array}{l}\text { Enfatizar acerca do } \\
\text { conhecimento do enfermeiro } \\
\text { em casos de pacientes com } \\
\text { acidente vascular encefálico, } \\
\text { identificando o que é, quais } \\
\text { os seus sintomas e formas } \\
\text { de como um profissional de } \\
\text { enfermagem deve atuar. }\end{array}$ & $\begin{array}{l}\text { Revisão } \\
\text { integrativa da } \\
\text { literatura. }\end{array}$ & $\begin{array}{l}\text { Deve-se buscar uma melhor adequação e } \\
\text { auxilio e estratégia de saúde com } \\
\text { consultas básicas e especializadas por } \\
\text { partes dos especialistas de enfermagem } \\
\text { para que isso, possa reduzir a taxa de } \\
\text { mortalidade. }\end{array}$ \\
\hline A9 & $\begin{array}{c}\text { Silva et al. } \\
\text { 2019a }\end{array}$ & $\begin{array}{l}\text { Cuidados de enfermagem à } \\
\text { certima de Acidente vascular }\end{array}$ & $\begin{array}{l}\text { Identificar na literatura } \\
\text { quais os cuidados de } \\
\text { enfermagem no atendimento } \\
\text { da vítima sequelado de } \\
\text { acidente vascular cerebral. }\end{array}$ & Revisão literária. & $\begin{array}{l}\text { Por meio deste estudo ficou evidente a } \\
\text { importância dos cuidados prestados pelo } \\
\text { enfermeiro e sua equipe na assistência ao } \\
\text { paciente vitima de AVC. Estudo sobre } \\
\text { diagnósticos de enfermagem são } \\
\text { necessários para que haja melhor } \\
\text { conhecimento cientifico na enfermagem. }\end{array}$ \\
\hline A10 & $\begin{array}{c}\text { Silva et al. } \\
\text { 2020b }\end{array}$ & $\begin{array}{l}\text { Prevalência e fatores de risco } \\
\text { associados ao acidente vascular } \\
\text { cerebral em pessoas com } \\
\text { hipertensão arterial: uma } \\
\text { análise hierarquizada. }\end{array}$ & $\begin{array}{l}\text { Analisar prevalência e } \\
\text { determinar hierarquicamente } \\
\text { fatores de risco associado ao } \\
\text { AVC em pessoas com } \\
\text { hipertensão arterial. }\end{array}$ & $\begin{array}{l}\text { Estudo seccional } \\
\text { analítico } \\
\text { realizado em 17 } \\
\text { unidades de } \\
\text { Saúde (UBS). }\end{array}$ & $\begin{array}{l}\text { A prevalência foi explicada por uma } \\
\text { hierarquia entre os fatores de risco, } \\
\text { evidenciando proximamente aqueles } \\
\text { classificados como modificáveis. }\end{array}$ \\
\hline
\end{tabular}

Fonte: Oliveira \& Maciel (2021).

O risco de AVE começa a aumentar com o passar dos anos, com idade igual ou superior aos 60 anos e dobra a cada década. Entre os principais fatores de risco, existem fatores não modificáveis que são: a hereditariedade, o sexo e a etnia, sendo o sexo masculino e a raça negra que apresentam maior incidência de AVE isquêmica. Enquanto nos fatores de risco modificáveis, se classificam a hipertensão arterial, sendo o principal deles, onde acarreta um aumento superior a três vezes na incidência em casos de AVE (Cruz, 2016).

A hipertensão arterial sistêmica é o fator de risco principal para o AVE, pois aumenta a chance de desenvolver a doença de três a quatro vezes, sendo considerada diretamente responsável por, pelo menos, metade dos casos relatados.

Já no AVCH predominam também os fatores de risco modificáveis para doença ateromatosa, sendo a hipertensão arterial um fator predominante, seguido pelo álcool. Como causa menos frequentes não ateromatosas de AVCH destaca-se a angiopatia amiloide no idoso, o uso de drogas de vasoativas (como a cocaína) e o uso de anticoagulantes, malformações vasculares e hemorragias em lesões tumorais subjacentes. O tabagismo, seguido de hipertensão arterial e o álcool na hemorragia subaracnóidea não traumática, é o fator de risco modificável principal (Silva et al. 2019a).

Hipertensão Arterial: Tem sido o principal fator de risco para AVE. O valor médio na população nos níveis de pressão é de 12 por 8, onde é preciso manter-se alerta quando há elevação inesperada do nível normal recomendado. A hipertensão arterial apresenta inúmeras alterações no nosso organismo, acelerando o processo de aterosclerose, além de levar a possíveis rupturas de um ou mais vasos sanguíneos, assim como uma isquemia (Galvão e Soares, 2016).

Doença Cardíaca em especial doenças que produzem arritmias podem contribuir para um AVE. Se o coração não bater direito, ocorre certa dificuldade para que o sangue alcance o cérebro, e também os outros órgãos, levando a uma isquemia. Isto pode ocorrer em situações como: arritmias, doença de Chagas, infarto do miocárdio, problemas nas válvulas do coração, dentre outras (Lima et al. 2016).

Colesterol: O colesterol total elevado, associado com o colesterol Lipoproteínas de alta densidade (HDL) baixo são fatores de risco para AVCI em ambos os sexos. Um dos principais fatores de risco para doenças vasculares, principalmente nos pacientes diabéticos que apresentam alta incidência de hipertensão arterial é o colesterol sérico, porque se associa positivamente ao enfarte cerebral. Quando seus níveis apresentam alterados, especialmente a fração Lipoproteínas de baixa densidade (LDL) (denominado colesterol ruim) que estão presentes em gorduras saturadas, ou seja, de origem animal, ou a 
redução dos níveis da fração HDL (denominado colesterol bom) estão relacionados com a formação das placas de aterosclerose (Oliveira et al. 2018).

Tabagismo: outro importante fator que age acelerando o processo de aterosclerose, contribuindo para a formação de sangue concentrado ou denso, e para o aumento na quantidade de glóbulos vermelhos, como também, aumentando o risco de hipertensão arterial. Em pesquisas realizadas com mulheres, foi observado um aumento de AVE hemorrágico, pelo tabagismo, causando hemorragia intracerebral e subaracnóidea por esse fator, sendo que com a diminuição ou após a cessação desse ato, diminui o risco de mortalidade sobre o AVE e doenças coronarianas (Rodrigues et al. 2019).

Uso excessivo de bebidas alcoólicas: Esse fator é relevante quando a quantidade de tempo e excessividade que é consumido são elevadas. Porque, através do álcool os níveis de colesterol se elevam, levando o indivíduo a uma maior pretensão à hipertensão arterial. O seu consumo excessivo é um fator de risco para todos os tipos de AVE, sendo o consumo moderado, uma redução desses riscos. O que influencia no grau de risco é o tipo de bebida alcoólica, sendo este efeito defensor mais consistente é encontrado para a ingestão de vinho, e em menor grau, para a cerveja, do que para outras bebidas (Silva et al. 2019a).

Sangue muito concentrado: Esse fator pode ocorrer, por exemplo, quando o indivíduo fica gravemente desidratado ou quando existe um aumento na quantidade dos glóbulos vermelhos, este último normalmente ocorre em pessoas que apresentam doenças pulmonares crônicas, ou em pessoas que vivem em grandes altitudes (Lima et al. 2016).

Diabetes Mellitus: uma patologia em que consiste o nível elevado de açúcar (glicose) no sangue, onde a medida de glicose no sangue é obtida pelo exame de glicemia. Sendo a glicemia devidamente controlada no sangue, o risco para o AVE é de menor gravidade, quanto aqueles que não são controlados. Essa patologia é relacionada a doenças de pequenos vasos, portanto o controle glicêmico nesses pacientes, em especial naqueles que já tiveram o primeiro episódio de AVE, deve ser o mais rigoroso possível (Zukerman, 2016).

Obesidade: Como um importante fator, age aumentando o risco de diabetes, seguido de hipertensão arterial e aterosclerose, assim, indiretamente, aumentando o risco para a ocorrência de AVE (Lima et al. 2016).

Anticoncepcionais hormonais: Sob pesquisas, esse se tornou um importante fator bem reconhecido para a ocorrência de AVE entre mulheres que fazem uso de pílula anticoncepcional, por causa da concentração dos hormônios, especialmente o estradiol. A ocorrência de AVE, com o efeito da pílula entre mulheres com mais de 35 anos é também maior, quanto naquelas com outros fatores de risco vascular como hipertensão arterial, obesidade, dislipidemia, tabagismo, algumas condições genéticas e enxaqueca com aura (Silva et al. 2020).

Sedentarismo: A falta de atividades físicas leva a muitas consequências graves à saúde, sendo um importante fator de risco para o AVE, pois o sedentarismo contribui para a obesidade, predispondo ao diabetes, consequentemente à hipertensão e o aumento do colesterol (Lima et al. 2016).

Idade: Quanto maior a idade, maior a probabilidade de ter um AVE, não excluindo a possibilidade de uma pessoa jovem ter chances. Pois os efeitos acumulativos do envelhecimento, associados ao aumento do número de fatores de risco, com o passar dos anos acrescem significativamente, contribuindo para o risco de enfarte e hemorragia cerebral. Estimando-se que o risco de AVE duplique em cada 10 anos depois dos 55 anos Raça: É mais frequente na raça negra e nos hispano-americanos (Silva et al. 2019a; Lima et al. 2016c).

Sexo: Homens com até 51 anos de idade tem maior pretensão do que as mulheres, sendo que depois desta idade, o risco, praticamente, é o mesmo para ambos. Em geral entre os homens há maior incidência de AVE do que nas mulheres. Porém, entre os 35 e os 44 anos o risco nas mulheres aumenta para a incidência de AVE, provavelmente sendo relacionada com a gravidez e o uso de contraceptivos orais (Silva et al. 2019a). 
Histórico Familiar: Estudos sobre pessoas na família que já tiveram AVE evidenciam que há aumento da susceptibilidade genética, representado pelo histórico positivo, está diretamente ligada ao desenvolvimento desse agravo, assim como infarto do miocárdio, ou doença vascular de membros, a probabilidade aumenta em ter um AVE.

Isto pode ocorrer pela transmissão de um único gene de doenças hereditárias raras, ou pela transmissão de fatores poligênicos como a facilidade de desenvolver hipertensão, diabetes, dislipidemia, que são determinados fatores de risco vascular, ou a susceptibilidade aos seus efeitos. As doenças monogênicas associadas ao AVE afetam pessoas mais novas, sobretudo são raras, associam- se a um risco muito elevado e têm diagnóstico molecular (Barnett e Spence, 2016).

A pressão arterial mesmo estando muito alta pode não apresentar nenhum sintoma, ficando assim por vários meses até anos, contudo podem ocorrer complicações comprometendo vasos do cérebro, do coração, dos olhos, dos rins e dos pés (Aleluia et al. 2017).

Em média $40 \%$ dos acidentes vasculares encefálicos e $25 \%$ dos infartos ocorridos em pacientes hipertensos conseguem tornar-se prevenidos com terapia anti-hipertensiva apropriada (Santana e Chun, 2017).

Os programas de nível nacional para detecção das doenças crônicas não transmissíveis como a hipertensão vêm cada vez mais discutidos como os tipos de programas que conseguem tornarem-se eficazes com a hipertensão. Tem sido imposta a melhora dos sintomas, o controle de peso, conduta de prática física, melhora na refeição (Nunes et al. 2017).

A alta prevalência de complicações microvasculares quando se descobre o diagnóstico, dá-se devido à demora do diagnóstico, pois ela pode ficar por um período assintomático, sendo que com o diagnóstico precoce e início do tratamento as complicações seriam mais difíceis de aparecerem (Silva et al. 2020b).

Os fatores de risco coronários documentados incluem o fumo, diabetes, hipercolesterolemia, hipertensão, sedentarismo, níveis de fibrinogênio elevados, e obesidade, particularmente a obesidade central. Os fatores de risco coronários predominam em populações menos favorecidas quanto ao desenvolvimento socioeconômico e educacional. Isso pode estar relacionado com falta de informação e menor acesso aos serviços de saúde (Aleluia et al. 2017)

A prevalência do tabagismo é igual para homens e mulheres, nos Estados Unidos. Os homens têm cessado mais o hábito de fumar nos últimos anos. Pelo fato de as campanhas de combate ao cigarro serem dirigidas mais ao público masculino, muitas mulheres tabagistas não se percebem estando no grupo de risco. Nos Estados Unidos, existe uma elevada prevalência de mulheres jovens tabagistas. O fumo, pelo menos, triplica o risco de infarto do miocárdio, mesmo nas mulheres antes da menopausa, e diminui a idade da menopausa em 1.5-2 anos. Parece existir, também, um efeito sinérgico do tabagismo e o uso de contraceptivos (Galvão e Soares, 2016).

Dentro de 2 anos de cessação do fumo, porém, foi observado uma diminuição de $24 \%$ no rico de mortalidade cardiovascular. Grandes tabagistas tendem a ter os mesmos riscos de apresentar infarto do miocárdio e doença coronária fatal que as mulheres, que nunca fumaram. Mesmo em pacientes com doença arterial coronária que cessaram o hábito de fumar tendem a ter um melhor prognóstico e menor índice de re-infarto (Silva et al. 2016c).

Aproximadamente $20 \%$ de todas as mortes por doenças cardiovasculares são atribuídas ao fumo. Estima-se que existem 22.6 milhões de mulheres tabagistas, as quais, quase $21.5 \%$ das mulheres são americanas. É notado, também, um aumento do hábito de fumar entre as mulheres brasileiras, constituindo importante fator de risco coronário (Galvão e Soares, 2016).

A prevalência dos fatores de risco aumenta com a idade, exceto o fumo, que tende a diminuir em prevalência. Assim, os fatores de risco são mais prevalentes nas mulheres em idade mais avançada, culminando com um maior risco de doença coronária nessa idade. As mulheres americanas mais jovens apresentam menos fatores de risco quando comparadas com homens ou com mulheres mais velhas (Silva et al. 2020b). 
Diabetes é um fator de risco mais forte para as mulheres do que para os homens, mesmo para as mulheres antes da menopausa. Mulheres com mais de 45 anos de idade são 2 vezes mais propensas a desenvolver diabetes do que homens. Uma vez ocorrido o infarto do miocárdio, diabetes mellitus é associado a um prognóstico menos favorável para mulheres do que para os homens. Após o infarto do miocárdio, mulheres diabéticas têm risco duplicado para apresentar recorrência do infarto e um risco quadruplicado de falência cardíaca quando comparadas aos não-diabéticos. Diabetes também contribui para um prognóstico mais desfavorável para as mulheres, após procedimentos de revascularização miocárdica (Galvão e Soares, 2016).

A hipertensão arterial sistêmica se tornou uma dificuldade para a saúde pública, devido sua alta confluência e associação com as doenças cardiovasculares, independentemente dos fatores de risco associado, como obesidade, sedentarismo e tabagismo (Silva et al. 2016c).

A HAS é uma doença não transmissível considerada uma síndrome multifatorial, ou seja, está associada a diversos fatores (Barnett e Spence, 2016). Segundo Dias et al. (2019), em um estudo elaborado no município de Pelotas no estado do Rio Grande do Sul, apenas com a população urbana, utilizando uma amostra de 1.968 indivíduos entre 20 a 69 anos de idade, obteve-se uma prevalência de 23,6\% da HAS. Ainda de acordo com os autores, entre os fatores associados, foi possível observar que de todos os indivíduos que participaram da pesquisa, $60 \%$ possuíam histórico familiar de doença hipertensiva; que as mulheres apresentaram 17\% a mais de probabilidade de apresentar HAS do que os homens; que a distribuição de HAS com relação à idade apresentava tendência positiva; que 53\% apresentaram sobrepeso ou obesidade, e $65 \%$ ingeriam bebidas alcoólicas em quantidades inferiores a $30 \mathrm{~g} / \mathrm{dia}$.

Silva et al. (2016c), em um estudo conduzido em São Luís no estado do Maranhão, utilizando uma amostra de 835 indivíduos com idade entre 18 a 94 anos, verificaram uma prevalência de HAS de 27,4\%, sendo a prevalência maior no sexo masculino $(31,1 \%)$, quando comparada ao sexo feminino $(24,2 \%)$ e que maiores prevalências de HAS foram observadas naqueles com escolaridade $12 \mathrm{e}<4$ anos, enquanto as menores prevalências foram observadas nos grupos com escolaridade intermediária.

Em um estudo realizado por Silva et al. (2016c), na Comunidade Quilombola Boqueirão, situada na zona rural do município de Vitória da Conquista, no Estado da Bahia, utilizou uma amostra de 213 adultos, de ambos os sexos, com a média de idade de 41 anos (DP \pm 17 ), sendo 59,2\% do sexo feminino e 40,8\% do sexo masculino. Os autores observaram que a prevalência de HAS foi de 38,5\% e que as variáveis escolaridade e renda permaneceram independentemente associadas à HAS, com maiores prevalências nos indivíduos de menor escolaridade e maior renda per capita.

A HAS é considerada um dos grandes problemas de saúde pública da atualidade, agravada por sua alta prevalência, no Brasil e no mundo, e diagnóstico frequentemente tardio. Esse agravo é caracterizado por apresentar um quadro clínico lento e assintomático, vários fatores de risco que agem simultaneamente, faltam na adesão ao tratamento, ou quando não tratada corretamente. Os altos índices de pressão arterial por um tempo prolongado podem ocasionar complicações no coração, rins, encéfalo e vasos sanguíneos, devido à agressão que o endotélio vascular sofre pela alta pressão nas artérias de pequeno porte (Paiva et al. 2016).

O aumento da carga da HAS foi um importante desafio para os sistemas de saúde, pois, durante muitos anos não havia a adoção de políticas específicas e amplas medidas de intervenção que facilitassem uma articulação de ações para prevenção, diagnóstico precoce, acompanhamento e tratamento dos hipertensos (Aleluia et al. 2017).

Diante dos marcos legais das políticas de saúde, em 1994, o Ministério da Saúde reorganizou essas políticas por meio do fortalecimento da atenção básica à saúde a partir da Estratégia Saúde da Família (ESF), com o intuito de prover a atenção básica à toda população de acordo com os princípios do Sistema Único de Saúde (SUS). Os profissionais que compõem a ESF são qualificados para identificar os casos que precisam de outros níveis da atenção à saúde, porém, a Atenção Primária à Saúde (APS), consegue dar suporte à maioria dos hipertensos (Cruz, 2016). 
A Unidade de APS trata o indivíduo como um todo pelo fato de estar inserida no contexto da prevenção, promoção e assistência, a fim de evitar danos muitas vezes incapacitantes. O cuidado pela APS favorece melhorias em termos de acesso, qualidade e continuidade da atenção, por integrar serviços e ações entre diversos níveis, ou no interior de um mesmo nível do sistema de saúde (Aleluia et al. 2017).

Os instrumentos da prática da APS como as consultas periódicas, grupos educativos e visitas domiciliares, são importantes para resolver grande parte das questões provocadas. Além de todo esse cuidado, o Ministério da Saúde, em 2001, propôs um Plano de Reorganização da Atenção à Hipertensão Arterial Sistêmica e ao Diabetes Mellitus, identificando a importância da atenção básica no tratamento desses agravos, realizada pelo modelo de atenção programática caracterizado como HIPERDIA, sistema de cadastramento e acompanhamento de hipertensos e diabéticos, que favorece desenvolver ações contínuas e englobando todos os pacientes.

A proposta do HIPERDIA recomenda ir além do binômio queixa-conduta, com o propósito de identificar os portadores assintomáticos, monitorar o tratamento, estabelecer vínculos entre equipe de saúde-pacientes-cuidadores e praticar a educação em saúde, a fim de incorporar a realidade do paciente nesse processo (Dias et al. 2019).

O crescimento das ações para com esse grupo deve ser preconizado, em razão do custo-benefício do controle da HAS em detrimento do custo para o tratamento dos seus agravos, uma vez que os gastos na saúde são diminuídos quando a intervenção é feita na atenção primária, e não nos outros níveis, havendo também maior possibilidade de promover uma melhor qualidade de vida para a população (Loft et al. 2017).

Os níveis de HDL-colesterol são maiores entre as mulheres do que entre os homens durante a vida, mesmo após a menopausa, havendo tendência à aproximação após os 60 anos de idade. Só um leve declínio dos níveis de colesterol HDL é observado na menopausa.

Os níveis LDL-colesterol são menores nas mulheres em idade mais jovem, quando comparados aos homens, aumentando progressivamente com a idade, particularmente nos anos após a menopausa, atingindo níveis maiores do que os dos homens na idade mais avançada. A elevação do colesterol LDL contribui para o aumento progressivo do colesterol-total com a idade entre as mulheres (Loft et al. 2017).

Portanto, após a menopausa, as várias frações lipídicas sofrem modificações, habitualmente com elevação dos níveis séricos da colesterolemia total, LDL-colesterol, triglicerídeos e Lipoproteína de densidade muito baixa (VLDL) - colesterol e com discreta e progressiva diminuição de HDL-colesterol.

A elevação de LDL-colesterol é condicionada por menor número de receptores para essas partículas e de VLDLcolesterol, por menor capacidade de sua transformação em VLDL remanescentes. Em relação à fração HDL-colesterol, as modificações são menos expressivas, ocorrendo pequena diminuição dos níveis circulantes em geral, correspondendo a menores níveis de apo A-I, provavelmente por haver menor transcrição dos mRNA para a síntese dessa apoproteína. Com essas alterações lipídicas ocorre aumento do risco para eventos coronários fatais e não-fatais. Esses achados parecem diretamente relacionados à menor síntese de estrógenos no período. Assim, os hormônios sexuais são potentes moduladores do metabolismo lipídico (Nunes et al. 2017).

Os efeitos dos progestágenos sobre o metabolismo lipídico são menos claros, mas sua atividade pode estar em parte determinada por sua ação androgênica. Por exemplo, sabe-se que progestágenos androgênicos diminuem níveis de VLDL e de triglicerídeos por aumentar o grau de seu catabolismo. Há, em geral, redução de HDL induzida por progestágenos, que pode estar ligada à maior atividade da lipase hepática (Galvão e Soares, 2016).

Durante o ciclo menstrual os níveis plasmáticos dos lípides circulantes sofrem influência das variações nas concentrações dos hormônios esteroides. Mulheres pré-menopausadas apresentam menores níveis de LDL.Existem controvérsias a respeito do papel da hipertrigliceridemia como fator de risco coronário para as mulheres, apesar de não ser 
considerado, em geral, como fator de risco para homens. No entanto, quando a hipertrigliceridemia é associada a baixos níveis de HDL-colesterol, essa combinação confere maior risco para mulheres do que para homens (Galvão e Soares, 2016).

Os sinais mais frequentes são caracterizados por fraqueza, dificuldade ao falar e para entender, adormecimento em apenas um lado do corpo, dificuldade para engolir, dificuldades para andar e enxergar, tontura, perda da força da musculatura no rosto que resulta em boca torta, cefaleia intensa, assim como perda da coordenação motora. Os sinais acontecem de forma súbita e podem ser únicos ou combinados (Barnett e Spence, 2016).

De forma geral, as manifestações de ambos os tipos de AVC podem ser semelhantes: podem surgir os sintomas durante o dia, de uma forma rápida e súbita, que, em poucos minutos ou poucas horas, pode atingir o máximo de intensidade, ou então os sintomas de AVE podem ocorrer durante o sono do paciente.

\section{Considerações Finais}

É de responsabilidade do profissional de enfermagem liderar grupos de educação voltados ao reconhecimento e estratégias de minimização da ocorrência de AVEs, os quais estabeleçam prioridades, como a necessidade de recorrer ao hospital.

Em um diagnóstico clínico de hemorragia intracerebral, deve-se distinguir as manifestações relativas ao advento, e as especificas da área cerebral que foi afetada. Podendo se destacar manifestações clínicas comuns como vômitos, cefaleias, e depressão do nível de consciência. Traumatismos cranianos e vasculites também são causas admitidas de hemorragias intracerebrais, destas, principalmente a depressão do nível de consciência, podem estar relacionadas com o tamanho do hematoma e com a mortalidade.

Os fatores de risco mais elevado de AVE que devem ser observados pela equipe de enfermagem são para as pessoas com hipertensão arterial sistêmica (HAS), ao contrário das pessoas normotensas onde pode ser observado uma menor incidência de AVE. Um resultado neurológico comum de um AVE na área motora do hemisfério afetado é a hemiplegia (paralisia de um lado do corpo. Ela ocorre no lado oposto ao lado da área cerebral afetada, pois, os nervos motores cruzam (decussação) ao nível do pescoço. Por exemplo, quando a área afetada motora do lado direito do cérebro sofre um AVE, a hemiplegia afeta o lado esquerdo do corpo. Imediatamente após o AVE, o lado afetado mostra flacidez e evoluiu para a espasticidade dos membros. Tipicamente, o membro superior é mais afetado do que o inferior.

Existe uma preocupação em salientar a importância da enfermagem na educação em saúde a fim de orientar e dar assistência à comunidade com enfoque preventivo e na busca de estratégias e metas para reabilitar os pacientes que apresentam sequelas da doença, bem como orientar as famílias e demais cuidadores quanto aos cuidados específicos relacionados ao AVE e sua progressão.

\section{Referências}

Aleluia, I. R. S., Medina, M. G., Almeida, P. F., \& Vilasbôas, A. L. Q. (2017). Coordenação do cuidado na atenção primária à saúde: estudo avaliativo em município sede de macrorregião do nordeste brasileiro. Ciência \& Saúde Coletiva, 22(6), p. 1845-1856.

Azevedo, D. K. L., Silva, C. M. P., \& Maia, A. L. (2021). O papel da gestão de enfermagem na implementação da meta de cirurgia segura: uma revisão de literatura. Research, Society and Development, 10(14), e584101422711, 2021 (CC BY 4.0) | ISSN 2525-3409 | DOI: http://dx.doi.org/10.33448/rsd-v $10 \mathrm{i} 14.22711$.

Bardin, L. (2012). Análise de conteúdo: a revisão de Laurence Bardin. Revista Eletrônica de Educação. Programa de Pós-graduação em Educação. 6(1), maio 2012. http://dx.doi.org/10.14244/\%2519827199291.

Barnett, H. J. M., \& Spence, J. D. (2016). Acidente vascular cerebral: prevenção, tratamento e reabilitação. 2. ed. Porto Alegre: Ed. AMGH.

Cruz, M. C. S. (2016). Terapia Ocupacional Na Reabilitação Pós-acidente Vascular Encefálico. 3 ed. São Paulo: Ed. Santos.

Dias, D. C., Alfieri, A. F., \& Battistella, B. L. (2019). Utilização de monofilamentos para avaliação sensorial em pacientes com sequela de Acidente Vascular Encefálico (AVE) - uma revisão sistemática. Revista Brasileira de Neurologia, 55(3), p. 22-28. 
Fernandes, A. M., Bruchêz, A., d'Ávila, A. A. F., Castilhos, N. C., \& Olea, P. M. (2018). Metodologia de pesquisa de dissertações sobre inovação: análise bibliométrica. Desafio Online, Campo Grande, 6(1), Jan./Abr. 2018. 141-159.

Galvão, R. R., \& Soares, D. (2016). Prevalência de hipertensão arterial e fatores associados em adultos: uma revisão na literatura brasileira. Revista APS, 19(1), p. 139-149.

Lima, A., Silva, A., Guerra, D., Barbosa, I., Bezerra, K., \& Oriá, M. (2016). Diagnósticos de enfermagem em pacientes com acidente vascular cerebral: revisão integrativa. Revista Brasileira de Enfermagem. 69(4), p. 785-792.

Loft, M.I., Martinsen, B., Esbensen, B., Mathiesen, L., Iversen, H., \& Poulsen, I. (2017). Fortalecendo o papel e as funções da equipe de enfermagem na reabilitação do Acidente Vascular Encefálico em pacientes internados: o desenvolvimento de uma intervenção complexa usando a roda de mudança de comportamento. Journal of the International Society For Quality In Health Care. Saúde Bem-estar, 12(2), p. 1-15.

Nunes, D. L. S., Santos, F. W. S., \& Lima, M. A. (2017). Cuidado de Enfermagem ao Paciente Vítima de Acidente Vascular Encefálico. Revista Brasileira de Ciências da Saúde, 21(1), p. 87-96.

Oliveira, A. K. S., Fernandes, A. F. L., Carvalho, G. F., Nascimento, L. K. A., Pellense, M. C. S., \& Santana, P. G. C. (2018). O papel do enfermeiro no cuidado a pacientes acometidos por acidente vascular encefálico. Revista Humano Ser - UNIFACEX, 3(1), p. 145-160.

Paiva, M. M., Dias, F. A., Molina, N. P. F. M., \& Tavares, D. M. S. (2016). Impacto da Hipertensão Arterial na qualidade de vida de idosos residentes na zona rural. Revista de Enfermagem e Atenção à Saúde, 5(1), p. 12-22.

Rodrigues, A. A., Paula, A. K., Santos, K. R. T., Aoyama, E. A., \& Souza, R. A. (2019). O conhecimento do enfermeiro e sua atuação no acidente vascular encefálico. Revista Brasileira Interdisciplinar de Saúde., 1(1), p. 55-61.

Santana, M. T. M., \& Chun, Y. S. C. (2017). Linguagem e funcionalidade de adultos pós Acidente Vascular Encefálico (AVE): avaliação baseada na Classificação Internacional de Funcionalidade, Incapacidade e Saúde (CIF). Revista da Sociedade Brasileira de Fonoaudiologia, 29(1), p. 255-284, 2017.

Silva, D., Melo, M., Duarte, E., \& Borges, A. (2019a). Cuidados de enfermagem à vítima de acidente vascular cerebral (AVC): Revisão integrativa. Revista Eletrônica Acervo Saúde, n. 36, p. e2156.

Silva, E., Borges, J., Moreira, T., \& Souza, A. (2020b). Prevalência e fatores de risco associados ao acidente vascular cerebral em pessoas com hipertensão arterial: uma análise hierarquizada. Revista de Enfermagem Referência, 5(3), p. 1-8.

Silva, T., Bomfim, C., Leite, T., Moura, C., Belo, N., \& Tomazi, L. (2016c). Hipertensão arterial e fatores associados em uma comunidade quilombola da Bahia, Brasil. Cadernos Saúde Coletiva, Rio de Janeiro, 24(3), p. 376-383.

Souza, M. T., Silva, M. D., Carvalho, R. (2010). Revisão integrativa: o que é e como fazer. Einstein. 2010; 8(1):102-6. https://www.scielo.br/j/eins/a/ZQTBkVJZqcWrTT34cXLjtBx/?format=pdf\&lang=pt.

Zukerman, E. (2016). Acidente Vascular Cerebral: Protocolos Gerenciados do Hospital Israelita Albert Einstein. Barueri: Edição 1; Editora Manole. ISBN: 9788520429488 